\title{
Endocystoscopy for colonic polyps: Is there a future for this diagnostic modality in routine practice?
}

\section{(ㄷ)(1) $\odot$}

\author{
Authors \\ Yasushi Sano, Mineo Iwatate \\ Institution \\ Gastrointestinal Center and Institute of Minimally Invasive \\ Endoscopic Care (iMEC), Sano Hospital, Hyogo, Japan \\ Bibliography \\ Endosc Int Open 2021; 09: E1012-E1013 \\ DOI 10.1055/a-1468-4414 \\ ISSN 2364-3722 \\ (C) 2021. The Author(s). \\ This is an open access article published by Thieme under the terms of the Creative \\ Commons Attribution-NonDerivative-NonCommercial License, permitting copying \\ and reproduction so long as the original work is given appropriate credit. Contents
}

\author{
may not be used for commercial purposes, or adapted, remixed, transformed or \\ built upon. (https://creativecommons.org/licenses/by-nc-nd/4.0/) \\ Georg Thieme Verlag KG, Rüdigerstraße 14, \\ 70469 Stuttgart, Germany \\ Corresponding author \\ Yasushi Sano, Chief, Gastrointestinal Center and Institution of \\ Minimally Invasive Endoscopic Care (iMEC), Sano Hospital, 2- \\ 5-1 Shimizugaoka, Tarumi-ku, Kobe, Hyogo 655-0031, Japan \\ Fax: 81-78-785-0077 \\ ys_endoscopy@hotmail.com
}

In this issue, Mori et al. reported that a brand-new Al-CADx system (EndoBRAIN-Plus, OLYMPUS) using endocytoscopy showed sufficient diagnostic capability in the discrimination of invasive cancer and its high specificity may contribute to a reduction in unnecessary surgeries for large adenomas. I have read this article with great interest. The history of endocytoscopy and CADx began with a pilot trial conducted on the esophagus by Kodashima et al [1]. Thereafter, there have been many reports concerning the usefulness thereof in the identification of tumors and invasion depth diagnosis when applied in colon [2-5]. We have great interest in a part of this paper by Mori, which indicated that it is possible to use Al diagnosis to check for the presence of invasive cancer in colorectal lesions $2 \mathrm{~cm}$ or larger that are susceptible to overtreatment. However, they wrote that, "Apparently, identification of the histologically worst area in a lesion is a prerequisite of using EndoBRAIN-Plus in an appropriate way. We did not assess how endoscopists identify these areas in the study, which will be an important research topic in the future." In other words, when actual EndoBRAIN-Plus-CADx is assumed, it is necessary to identify the area of interest of the deepest part of the cancer infiltration in the lesion. Therefore, endoscopists need to have sufficient understanding of the fundamental diagnosis of colorectal lesions along with the methods of approaching them, with beginners left with the question of how this is possible. This approach suggests that, for the time being, it is realistic to apply CADx as an educational training tool to support beginners in getting better at fundamental diagnostic endoscopy. Also, because CADx cannot show the processes that led to a diagnosis (black box diagnosis), research should also be conducted into whether it is possible to actually provide this kind of education to beginners in a logical manner.

The majority of original papers on the subject of endocytoscopy come out of Japan. There are few reports from countries other than Japan, so this area has not yet become pervasive $[6,7]$. However, endocytoscopy can be sufficiently applied in everyday clinical applications. Our hospital has been using it for everyday clinical applications since 2016. We confirmed insertion time for the endocytoscope reaching the cecum was acceptable compared with others (EC 5.5 min vs. Others $6.4 \mathrm{~min}$ ). Because the magnification power of endocytoscopy ranges from standard to ultra-high, we can obtain a standard image with $\mathrm{NBI}$ and the cellular image with dye staining at the same time ( $\mathbf{F i g} \mathbf{1}$ ). However, because endoscopic diagnosis with standard magnification also provides sufficient diagnostic accuracy, there are very few cases in which routine clinical applications require ultra-high magnification. The appeal of endocytoscopy is that it is well designed for CADx, given its ability to easily yield uniform images with a contact approach to colorectal lesions. Naturally, another appealing point is its ability to observe cellular-level information in real time. Dissemination of CADx before sufficient research has been performed into the clinical effectiveness of ultra-high magnification, which is the original objective of endocytoscopy, could reduce the enthusiasm that younger researchers have for diagnostic endoscopy with ultra-high magnification. Shouldn't we not only focus on what is available now, but also have more discussions about the significance and appeal of ultra-high magnification endocytoscopy in the future? We believe that surely, there exists a variety of yet-unknown information still to be discovered. 

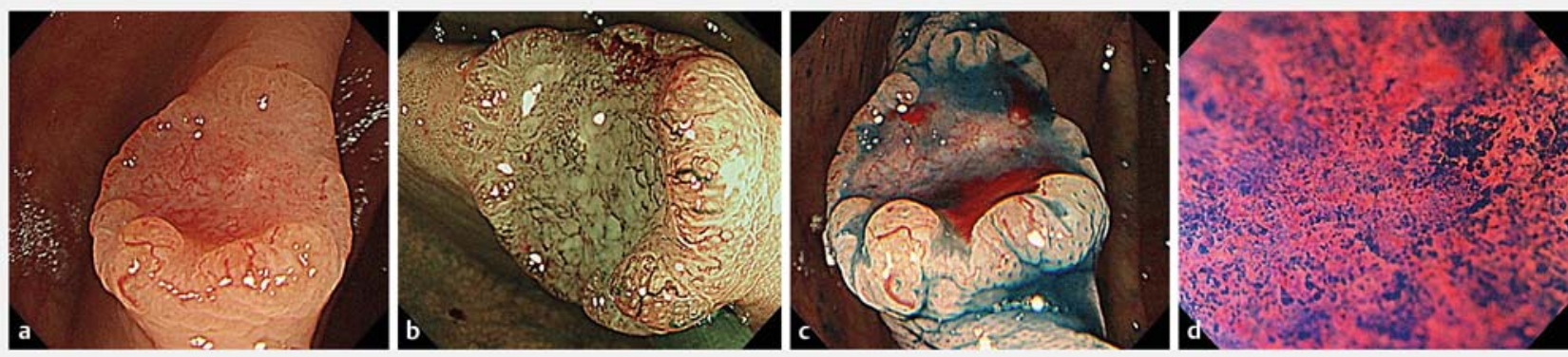

- Fig. 1 Simultaneous observation of a colon polyp (IIa + Ilc, $8 \mathrm{~mm}$, T1b adenocarcinoma) under WLI, NBI, and chromoendoscopy using endocytoscopy. a WLI. b NBI (JNET Type 3). c Chromoendoscopy. d Cellular image (EC 3b, × 520).

\section{Competing interests}

The authors declare that they have no conflict of interest.

\section{References}

[1] Kodashima S, Fujishiro M, Takubo K et al. Ex vivo pilot study using computed analysis of endo-cytoscopic images to differentiate normal and malignant squamous cell epithelia in the oesophagus. Dig Liver Dis 2007; 39: 762-766

[2] Mori Y, Kudo S-e, Wakamura K et al. Novel computer-aided diagnostic system for colorectal lesions by using endocytoscopy (with videos). Gastrointest Endosc 2015; 81: 621-629
[3] Mori Y, Kudo SE, Chiu PW et al. Impact of an automated system for endocytoscopic diagnosis of small colorectal lesions: An international web-based study. Endoscopy 2016; 48: 1110-1118

[4] Misawa M, Kudo SE, Mori Y et al. Characterization of colorectal lesions using a computer-aided diagnostic system for narrow-band imaging endocytoscopy. Gastroenterology 2016; 150: 1531-1532.e3

[5] Takeda K, Kudo S, Mori Y et al. Accuracy of diagnosing invasie colorectal cancer using computer-aided endocytoscopy. Endoscopy 2017; 49: 798-802

[6] Meroni E, Gatteschi B, Fasoli A et al. Detection of tissue abnormalities in normal mucosa surrounding colorectal cancer using an endocytoscopy system. Endoscopy 2007; 39: 369-370

[7] Neumann H, Vieth M, Neurath MF et al. Endocytoscopy allows accurate in vivo differentiation of mucosal inflammatory cells in IBD: a pilot study. Inflamm Bowel Dis 2013; 19: 356-362 